\title{
Phase II study of palliative S-1 in combination with cisplatin as second-line chemotherapy for gemcitabine-refractory pancreatic cancer patients
}

\author{
HYUN JUNG KIM ${ }^{1}$, JINA YUN ${ }^{1}$, HAN JO KIM ${ }^{4}$, KYOUNG HA KIM ${ }^{3}$, SE HYUNG KIM ${ }^{1}$, \\ TAE HOON LEE ${ }^{5}$, SANG-CHEOL LEE ${ }^{3}$, SANG BYUNG BAE ${ }^{4}$, CHAN KYU KIM ${ }^{1}$, NAM SU LEE ${ }^{3}$, \\ JONG HO MOON ${ }^{2}$, SANG HEUM PARK $^{5}$, KYU TAEK LEE ${ }^{4}$, SEONG KYU PARK ${ }^{1}$, \\ JONG-HO WON ${ }^{3}$, HEE SOOK PARK ${ }^{4}$ and DAE SIK HONG ${ }^{1}$

\begin{abstract}
${ }^{1}$ Department of Internal Medicine, Division of Hematology and Oncology; ${ }^{2}$ Department of Gastroenterology, Soonchunhyang University Bucheon Hospital, Bucheon 420-767; ${ }^{3}$ Department of Internal Medicine, Division of Hematology and Oncology, Soonchunhyang University Seoul Hospital, Seoul 140-743; ${ }^{4}$ Department of Internal Medicine, Division of Hematology and Oncology; ${ }^{5}$ Department of Gastroenterology, Soonchunhyang University Cheonan Hospital, Cheonan 330-721, Republic of Korea
\end{abstract}

Received January 2, 2012; Accepted March 2, 2012

DOI: $10.3892 / 01.2012 .637$

\begin{abstract}
In this study, we examined the efficacy and toxicity of S-1 with cisplatin as a second-line palliative chemotherapy for gemcitabine-refractory pancreatic cancer patients. Patients who had been previously treated with gemcitabine-based chemotherapy as palliative first-line chemotherapy received $\mathrm{S}-1 /$ cisplatin [body surface area (BSA) $<1.25 \mathrm{~m}^{2}, \mathrm{~S}-140 \mathrm{mg} /$ day; $B S A \leq 1.25$ to $<1.5 \mathrm{~m}^{2}, 50 \mathrm{mg} /$ day; $B S A \geq 1.5 \mathrm{~m}^{2} 60 \mathrm{mg} /$ day, orally, bid, daily on days 1-14 followed by a 7-day washout and cisplatin $60 \mathrm{mg} / \mathrm{m}^{2} /$ day intravenously on day 1] every three weeks. The enrollment of 32 patients was planned, but the study was terminated early, prior to the first stage, following the enrollment of 11 patients. The median age of the patients was 56 (range, 42-74) years. Nine patients had a performance status (PS) of one. In total, there were 21 chemotherapy cycles and the median treatment duration was 21 (range, 7-96) days. Of the 11 patients, five could not be evaluated due to discontinuation prior to the response evaluation. One of the six evaluable patients achieved stable disease (9.1\% in intention to treat analysis and $16.7 \%$ in per-protocol analysis), while five had progressive disease. Grade 3-4 hematological toxicities were anemia in one, neutropenia in one and thrombocytopenia in one cycle. Grade 3-4 nonhematological toxicities were fatigue in three, nausea in four, anorexia in two, diarrhea in one and peripheral neuropathy in two cycles. With
\end{abstract}

Correspondence to: Dr Dae Sik Hong, Department of Internal Medicine, Division of Hematology and Oncology, Soonchunhyang University Bucheon Hospital, 170 Chomaru-ro, Wonmi-gu, Bucheon 420-767, Republic of Korea

E-mail: dshong@schmc.ac.kr

Key words: pancreatic cancer, S-1, cisplatin, gemcitabine-refractory a median follow-up period of 8.9 (range, 3.2-11.3) months, the median time to progression was 44 days [ $95 \%$ confidence interval (CI) 25.4-62.6] and the median overall survival was 81 days (95\% CI 9.3-152.7). Combination chemotherapy with $\mathrm{S}-1$ and cisplatin as applied in this study did not result in promising antitumor activity, a high degree of toxicity and poor compliance.

\section{Introduction}

The prognosis of patients with advanced pancreatic carcinoma is extremely poor despite numerous trials with palliative chemotherapy or radiotherapy. Systemic chemotherapy with gemcitabine has been the standard chemotherapy for advanced pancreatic cancer since the mid-1990s (1). However, there is no standard second-line chemotherapeutic drug in cases refractory to or recurring following gemcitabine therapy. The median survival rate with best supportive care in patients who have failed gemcitabine therapy is approximately two months $(2,3)$. Approximately half of patients with gemcitabine-pretreated disease may be candidates for further treatment. Data supporting the use of second-line therapy compared with best supportive care are lacking. Although there have been reports of clinical trials of second-line therapy in advanced pancreatic cancer, most of these have been published in abstract form with a small number of patients. Therefore, there is a continuing need for clinical trials with a new agent for advanced pancreatic cancer in cases of gemcitabine failure.

$\mathrm{S}-1$ is a fourth-generation oral fluoropyrimidine that has been reported to be active with tolerable toxicity against gemcitabine-refractory pancreatic cancer (4-6) and chemotherapy-naïve pancreatic cancer $(7,8)$, although most of the studies have been case reports or retrospective studies. The superior effect of combination therapy with cisplatin compared with 5-fluorouracil (5-FU) monotherapy has been 
demonstrated in advanced pancreatic cancer $(9,10)$. Thus, we conducted the present phase II study to investigate the feasibility and efficacy of S-1 in combination with cisplatin as palliative chemotherapy for gemcitabine-refractory advanced pancreatic cancer patients.

\section{Patients and methods}

Ethics. This was a prospective multicenter study. All patients provided written informed consent. In total, three centers participated. This study was approved by the Institutional Review Board (IRB) of each center and was conducted in accordance with the Declaration of Helsinki.

Patients. The inclusion criteria for this study were: i) histologically or cytologically proven pancreatic adenocarcinoma and unresectable locally advanced or metastatic disease; ii) at least one measurable lesion according to Response Evaluation Criteria in Solid Tumors (RECIST) (11); iii) prior chemotherapy with gemcitabine-based palliative chemotherapy; iv) the ability to take oral medications; v) age, $>18$ years; vi) an Eastern Cooperative Oncology Group (ECOG) performance status (PS) of 0-2; vii) adequate bone marrow function (white blood cell count $\geq 4,000 / \mathrm{mm}^{3}$, neutrophil count $\geq 2,000 / \mathrm{mm}^{3}$ and platelet count $\geq 100,000 / \mathrm{mm}^{3}$ ); viii) adequate renal function [serum creatinine level $\leq 1.5 \mathrm{mg} / \mathrm{dl}$ or creatinine clearance level (Ccr) $\geq 50 \mathrm{ml} / \mathrm{min}$ ]; ix) adequate liver function [total bilirubin $\leq 3 \mathrm{x}$ UNL (if due to underlying liver metastasis, then total bilirubin may be $\leq 5 \mathrm{x}$ UNL); aspartate transaminase (AST) and/or alanine transaminase (ALT) $\leq 2.5 x$ UNL (if liver function abnormalities were due to underlying liver metastasis, then AST and/or ALT may be $\leq 5 x$ UNL)].

The exclusion criteria for this study were patients who: i) had received chemotherapy or radiotherapy within 3 weeks; ii) had previously received an oral fluoropyrimidine; iii) had central nervous system metastases; iv) had an active infection or uncontrolled concurrent medical illness; v) had a history of other malignancies; vi) were pregnant or lactating; vii) had severe neurological impairment, a mental disorder or any severe drug-induced allergy.

Treatment protocol. S-1 [body surface area (BSA) $<1.25 \mathrm{~m}^{2}$, $40 \mathrm{mg} ; \mathrm{BSA} \leq 1.25$ to $\left.<1.5 \mathrm{~m}^{2}, 50 \mathrm{mg} ; \mathrm{BSA} \geq 1.5 \mathrm{~m}^{2}, 60 \mathrm{mg}\right]$ was administered orally twice daily, following breakfast and dinner, for 14 consecutive days, followed by seven days of rest. Cisplatin $\left(60 \mathrm{mg} / \mathrm{m}^{2}\right)$ was administered as a $60-\mathrm{min}$ intravenous infusion on day 1 with adequate hydration. The treatment courses were repeated every three weeks. Antiemetic prophylaxis, including aprepitant, a 5-HT3 antagonist and dexamethasone, was used. Prophylactic myeloid growth factors were not administered prior to the first cycle.

Dose modification. Modifications to the S-1 or cisplatin dose were made in patients who had any of the following: a leukocyte count $<1.0 \times 10^{3} / \mu 1$, a neutrophil count $<500 / \mu 1$, a platelet count $<2.5 \times 10^{4} / \mu 1$, grade 3-4 febrile neutropenia or grade 3-4 non-hematological toxicity, based on the most severe grade of toxicity that had occurred during the previous cycle. Treatment was delayed for up to three weeks in patients with persistent symptomatic toxicity, absolute neutrophil counts $<1,500 / \mu 1$ or platelet counts $<100,000 / \mu 1$. The dose of $S-1$ was decreased in a stepwise manner by up to two levels as follows: BSA $<1.25 \mathrm{~m}^{2}$, from 40 to 25 and $20 \mathrm{mg} / \mathrm{dose}$; BSA $\geq 1.25$ to $<1.5 \mathrm{~m}^{2}$, from 50 to 40 and $25 \mathrm{mg} / \mathrm{dose}$; BSA $\geq 1.5 \mathrm{~m}^{2}$, from 60 to 50 and $40 \mathrm{mg} /$ dose. Additionally, the dose of cisplatin was decreased according to the serum Ccr as follows: $\mathrm{Ccr} \geq 60 \mathrm{ml} / \mathrm{min}$, no reduction; Ccr $>40 \mathrm{ml} / \mathrm{min}$ to $<60 \mathrm{ml} / \mathrm{min}$, reduced to $30 \mathrm{mg} / \mathrm{m}^{2}$; Ccr $<40 \mathrm{ml} / \mathrm{min}$, administration of cisplatin was stopped. Treatment was continued until signs of disease progression or unacceptable toxic effects developed or until a patient refused further treatment.

Pretreatment evaluation. Baseline laboratory analyses [blood cell count, serum creatinine, bilirubin, AST, ALT, alkaline phosphatase, lactic dehydrogenase and carbohydrate antigen (CA) 19-9] were performed within one week of starting the first cycle of therapy and tumor status was assessed using computed tomography (CT) within 4 weeks.

Assessment of efficacy and toxicity. Tumor assessments, using CT of the lesions, abdomen, pelvis and/or chest, were performed at baseline and repeated every 3 cycles using RECIST (11). The tumor marker CA 19-9 was checked every three cycles. A physical examination, including weight and toxicity assessments, ECOG performance status, complete blood count and blood chemistry, was performed prior to each cycle. Toxicity was graded according to National Cancer Institute Common Toxicity Criteria (NCI-CTC), version 3.0. Treatment-related mortality (TRM) was defined as mortality that occurred within 30 days of treatment initiation.

Statistical analysis. The primary endpoint was response rate and the secondary endpoints were safety, time to progression (TTP), disease control rate and overall survival (OS). The sample size in this trial was calculated to reject a $5 \%$ response rate in favor of a target response rate of $20 \%$ with a significance level of 0.05 and a power of $80 \%$, using Simon's optimal two-stage design. In the initial stage, in total, 10 evaluable patients were to be entered and evaluated for a response. If there was no response, accrual was to be terminated. If any response was observed in the first stage, then 19 additional patients were to be entered in the second stage, to achieve a target sample size of 29 evaluable patients. Further assessment of the regimen was thought to be necessary if more than three responses were observed in the 29 patients. Considering a withdrawal rate of $10 \%$, the total target number was calculated to be 32 patients.

Assessment of the response rate was performed using the intention to treat (ITT) and per-protocol (PP) analyses. TTP and OS were calculated using the Kaplan-Meier method. Survival curves were compared using the log-rank test. Tests were two sided and $\mathrm{p}<0.05$ was considered to indicate a statistically significant result. TTP was calculated from the date therapy was initiated to the date of disease progression, mortality or final follow-up. OS was calculated from the date therapy was initiated to the date of mortality or final follow-up.

\section{Results}

Patient characteristics. Between October 2009 and June 2010, in the stage I analysis, 11 patients were enrolled in this 
Table I. Patient characteristics $(n=11)$.

\begin{tabular}{|c|c|c|}
\hline Characteristics & No. & $\%$ \\
\hline \multicolumn{3}{|l|}{ Gender } \\
\hline Male & 7 & 63.6 \\
\hline Female & 4 & 36.4 \\
\hline \multicolumn{3}{|l|}{ Age, years } \\
\hline Median (range) & $56(42-74)$ & \\
\hline \multicolumn{3}{|c|}{ Performance status (ECOG) } \\
\hline $0-1$ & 9 & 81.8 \\
\hline 2 & 2 & 18.2 \\
\hline \multicolumn{3}{|l|}{ CA 19-9 level } \\
\hline Within normal range & 5 & 45.5 \\
\hline$>$ Normal & 6 & 54.5 \\
\hline \multicolumn{3}{|c|}{ Location of primary tumor } \\
\hline Head & 6 & 54.5 \\
\hline Body & 2 & 18.2 \\
\hline Tail & 3 & 27.3 \\
\hline \multicolumn{3}{|l|}{ Disease status } \\
\hline Locally advanced & 2 & 18.2 \\
\hline Distant metastases & 9 & 81.8 \\
\hline \multicolumn{3}{|c|}{ Sites of distant metastases } \\
\hline Liver & 7 & - \\
\hline Lymph node & 3 & - \\
\hline Lung & 5 & - \\
\hline
\end{tabular}

ECOG, Eastern Cooperative Oncology Group; CA, carbohydrate antigen.

Table II. Response rate of S-1 and cisplatin for gemcitabinerefractory pancreatic cancer.

\begin{tabular}{lccc}
\hline & $\begin{array}{c}\text { No. of patients } \\
(\mathrm{n}=11)\end{array}$ & $\begin{array}{c}\text { ITT } \\
(\%)\end{array}$ & $\begin{array}{c}\text { analysis } \\
(\%)\end{array}$ \\
\hline Complete response & 0 & 0 & 0 \\
Partial response & 0 & 0 & 0 \\
Stable disease & 1 & 9.1 & 16.7 \\
Not evaluated & 5 & - & - \\
Response rate & 0 & 0 & 0 \\
Disease control rate & 1 & 9.1 & 16.7 \\
\hline
\end{tabular}

ITT, intention to treat; PP, per-protocol.

prospective study. The median age of the patients was 56 (range, 42-74) years. The male:female ratio was 7:4. Of the 11 patients, nine had a PS of one and two had a PS of two when enrolled. There were six $(54.5 \%)$ cases of primary tumors in the head, two (18.2\%) cases in the body and three $(27.3 \%)$ in the tail portion of the pancreas. Of the 11 patients, nine $(81.8 \%)$ had distant metastases, while the remaining two (18.2\%) had locally advanced disease. Six (54.5\%) patients had elevated CA 19-9 levels when enrolled (Table I).
A

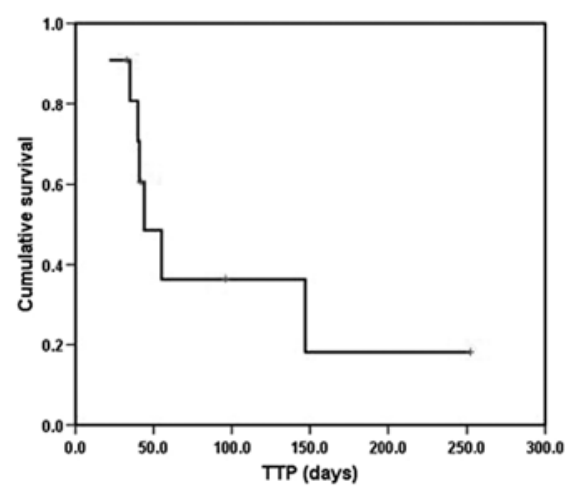

B

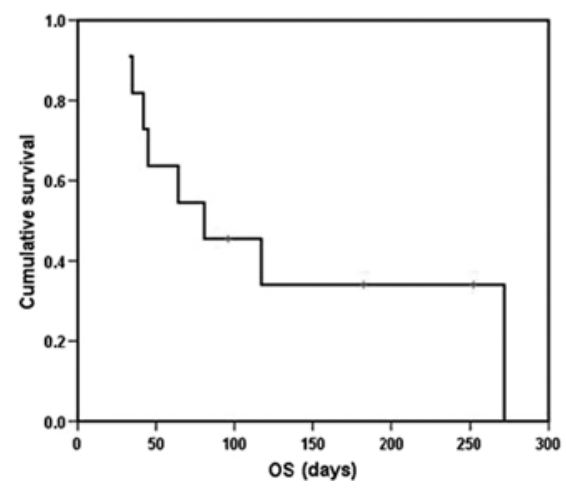

Figure 1. Kaplan-Meier curves for (A) the time to prognosis (TTP) and (B) overall survival (OS).

Delivery of drugs. In total, 21 cycles of therapy were administered, with a median of 1.5 (range, 1-5) cycles per patient and a median treatment duration of 21 (range, 7-96) days. The average relative dose intensities of S-1 and cisplatin were 0.98 and 0.91 , respectively. Dose reduction for $\mathrm{S}-1$ was required in one patient (two cycles) due to non-hematological toxicity, including diarrhea and fatigue. Dose reduction for cisplatin was required in two patients (three cycles) due to nausea and vomiting in one case (two cycles) and peripheral neuropathy in the other case (one cycle).

Tumor responses. Of the 11 patients enrolled in this study, six were evaluable in terms of treatment response. Five patients could not be evaluated for the following reasons: three withdrew consent due to therapy-related toxicities and worsening of their general condition and two died prior to the response evaluation. None of the six evaluable patients achieved a complete or partial response. Only one patient $(9.1 \%$ by ITT analysis and $16.7 \%$ by PP analysis) achieved stable disease and five had progressive disease (Table II).

Survival (TTP and OS). The patients were evaluable for the survival analysis. With a median follow-up of 8.9 (range, 3.2-11.3) months, the median TTP was 44 days [95\% confidence interval (CI) 25.4-62.6] and the median OS was 81 days (95\% CI 9.3-152.7). Kaplan-Meier curves for TTP and OS are shown in Fig. 1.

Toxicities. Grade 3-4 hematological toxicities included anemia in one cycle, neutropenia in one cycle and thrombocytopenia in one cycle. Grade 3-4 non-hematological toxicities included 
Table III. Adverse effects of S-1 and cisplatin for gemcitabinerefractory pancreatic cancer.

\begin{tabular}{lcc}
\hline $\begin{array}{l}\text { NCI-CTC } \\
\text { Grade 3-4 toxicities }\end{array}$ & $\begin{array}{c}\text { Per cycle } \\
\text { no. }(\%) \mathrm{n}=21\end{array}$ & $\begin{array}{c}\text { Per patient } \\
\text { no. }(\%) \mathrm{n}=21\end{array}$ \\
\hline $\begin{array}{l}\text { Hematologic toxicity } \\
\text { Neutropenia }\end{array}$ & $1(4.8)$ & $1(9.1)$ \\
Anemia & $1(4.8)$ & $1(9.1)$ \\
Thrombocytopenia & $1(4.8)$ & $1(9.1)$ \\
Non-hematologic toxicity & & \\
Nausea & $4(19.0)$ & $3(27.3)$ \\
Vomiting & $1(4.8)$ & $1(9.1)$ \\
Diarrhea & $1(4.8)$ & $1(9.1)$ \\
Fatigue & $3(14.3)$ & $3(27.3)$ \\
Anorexia & $2(9.5)$ & $2(18.2)$ \\
Peripheral neuropathy & $2(9.5)$ & $1(9.1)$ \\
\hline
\end{tabular}

NCI-CTC, National Cancer Institute Common Toxicity Criteria.

fatigue in three cycles, nausea in four cycles, anorexia in two cycles, diarrhea in one cycle and peripheral neuropathy in two cycles (one patient). There were two TRMs. The cause of mortality in the first case was septic shock associated with grade 3 neutropenia following the first cycle; in the second case, the patient died suddenly without documented cause following the second cycle. These toxicities are shown in Table III. This study was terminated early, prior to the first stage, without reaching 10 response-evaluable patients due to severe toxicity, including TRM, and poor compliance, by agreement of the investigators and the IRB.

\section{Discussion}

Pancreatic cancer is the fourth most common cause of cancer-related mortality in the US (12). In Korea, the incidence of this disease has increased. In 2009, the disease ranked 9th in incidence in Korea according to an annual report of cancer statistics; for cancer-related mortality, the disease ranked 5 th $(5.8 \%$ of the total). The prognosis of locally unresectable or metastatic pancreatic cancer remains extremely poor. Gemcitabine monotherapy or gemcitabine-based combination therapy, according to PS, has been the standard systemic therapy for advanced pancreatic cancer. FOLFIRINOX has been recommended as a first-line therapy with gemcitabine monotherapy or a gemcitabine-containing double regimen, based on a published phase III trial in which patients with metastatic pancreatic cancer showed marked improvements in median progression-free survival (PFS) (6.4 vs. 3.4 months; $\mathrm{p}<0.0001)$ and median OS (10.5 vs. 6.9 months; $\mathrm{p}<0.001)$ (13).

While first-line therapy has been established in advanced pancreatic cancer, there is no consensus with regards to a second-line therapy for advanced pancreatic cancer, particularly in gemcitabine-refractory cancer. It is difficult to conduct a clinical trial for second-line chemotherapy in advanced pancreatic cancer due to the rapidly progressive nature of the general condition and the lack of agents active in pancreatic cancer. However, it has been reported that $55-60 \%$ of patients had a relatively good PS following the failure of first-line therapy; thus, physicians should consider second-line therapy in such patients (14).

The results of previous studies concerning oxaliplatin (15), ralitrexed (16), paclitaxel (17) and pemetrexed (18) monotherapies in the second-line treatment of pancreatic cancer have revealed modest antitumor effects with no survival benefit. Studies have also reported combination chemotherapeutic regimens as second-line therapies for advanced pancreatic cancer. A representative study of second-line chemotherapy in pancreatic cancer is the CONKO-003 trial. In a preliminary report from the CONKO-003 trial, the use of second-line chemotherapy was compared with best supportive care (2). The study revealed the benefit of combination therapy with oxaliplatin, 5-FU and leucovorin as a second-line therapy compared with 5-FU and leucovorin (19). There have been other studies concerning combination chemotherapy for second-line therapy in pancreatic cancer with biological agents. However, the results are generally modest and preliminary $(20,21)$.

$\mathrm{S}-1$, an oral agent, consists of a mixture of tegafur, 5-chloro2,4-dihydroxypyridine and potassium oxonate at a molar ratio of 1:0.4:1. The antitumor effect of S-1 in advanced pancreatic cancer as a first- or second-line therapy has been reported in Japan (8,22-24). Generally, the antitumor effect was promising and the toxicity was tolerable in these studies. In view of the favorable toxicity profile of S-1 monotherapy, its combination with other agents may improve therapeutic results.

The addition of cisplatin offers the possibility of a synergistic antitumor effect, beyond that observed with S-1 monotherapy. Cisplatin combined with 5-FU appears to be promising in metastatic pancreatic carcinoma, with a $26 \%$ response rate and a median survival rate of 7 months in a phase II trial (10). In a randomized trial comparing 5-FU with 5-FU plus cisplatin, FU-cisplatin was found to be superior to FU in terms of response and PFS, but not OS $(9,10)$. The combination of S-1 and cisplatin has also been adopted in advanced gastric cancer, based on previous studies which revealed that combination therapy with S-1 and cisplatin had promising effects with tolerable toxicity $(25,26)$.

Although this study was conducted based on published data similar to those above, the results were disappointing compared with those of previous studies concerning monotherapy in advanced pancreatic cancer as a second-line therapy. The cause of the poor response and poor compliance may have been the rapid worsening of the general condition of the patients. In contrast to our results, Togawa et al (27) revealed that S-1 with cisplatin had promising effects in patients who failed postoperative gemcitabine treatment for pancreatic cancer. In that study, the dose of cisplatin was $40 \mathrm{mg} / \mathrm{m}^{2}$ and it was administered on day 8 every five weeks, to avoid the severe toxicity of cisplatin. Additionally, the group of patients had relapsed following postoperative gemcitabine treatment, unlike our patients who failed first-line palliative chemotherapy containing gemcitabine. These patients may have had a relatively good PS and maintained a good general condition relatively longer than those in our group. Additionally, in their group, there was no patient with previous exposure to cisplatin, unlike our group. These factors may explain the differences between our results and those of Togawa et al (27). 
In this study, one patient achieved stable disease. However, this patient experienced severe adverse events, including anorexia and nausea, and ultimately did not undergo more than 5 cycles. In the present study, we observed several types of hematological and non-hematological severe adverse events, including anorexia, nausea, fatigue, peripheral neuropathy and cytopenia. These adverse events resulted in poor compliance. Two patients had moderate toxicities, including nausea and anorexia; however, even these patients were reluctant to undergo further chemotherapy. The general fragility of the patients may have contributed to their poor compliance.

We also observed TRM in two patients. One experienced sepsis with severe neutropenia following the first cycle. Another patient succumbed to the disease suddenly following the second cycle, complaining of abdominal pain. The cause of mortality was not certain, but a thromboembolic event may have been the cause. These adverse events resulted in the discontinuation of the second-line therapy in these vulnerable patients. This issue should be considered in designing future clinical studies of advanced pancreatic cancer patients.

In the present study, we observed that the advanced pancreatic cancer patients progressed rapidly and that the general condition of the patients often deteriorated rapidly to perform additional chemotherapy cycles. Thus, prospective studies of palliative second-line therapy in patients with pancreatic cancer using combinations of novel or biological agents should consider the expectation for the worsening of the PS of the patients. Additionally, patients with good prognostic factors, as suggested by Nakachi et al (3), including a good PS, lower serum C-reactive protein levels and no peritoneal dissemination, should be considered as initial candidates for second-line chemotherapy in advanced pancreatic cancer.

In conclusion, this prospective combination chemotherapy study of S-1 and cisplatin did not demonstrate promising antitumor activity. Additionally, moderate toxicity profiles, with two cases of TRM, and poor compliance were observed in patients with advanced pancreatic cancer. In future studies, dose and schedule modification, as well as patient selection, are necessary for the precise evaluation of the effects of S-1 plus cisplatin on pancreatic cancer.

\section{References}

1. Burris HA III, Moore MJ, Andersen J, et al: Improvements in survival and clinical benefit with gemcitabine as first line therapy for patients with advanced pancreas cancer: a randomized trial. J Clin Oncol 15: 2403-2413, 1997.

2. Oettle H, Pelzer U, Stieler J, Hilbig A, et al: Oxaliplatin/folinic acid/5-fluorouracil [24h] (OFF) plus best supportive care versus best supportive care alone in second-line therapy of gemcitabinerefractory advanced pancreatic cancer (CONKO 003). J Clin Oncol 23 (Suppl): 4031, 2005.

3. Nakachi K, Furuse J, Ishii H, Suzuki E and Yoshino M: Prognostic factors in patients with gemcitabine-refractory pancreatic cancer. Jpn J Clin Oncol 37: 114-120, 2007.

4. Morizane C, Okusaka T, Furuse J, et al: A phase II study of S-1 in gemcitabine-refractory metastatic pancreatic cancer. Cancer Chemother Pharmacol 63: 313-319, 2009.

5. Morizane C: A case of gemcitabine-refractory pancreatic cancer responsive to second-line chemotherapy using S-1. Jpn J Clin Oncol 37: 973, 2007.

6. Todaka A, Fukutomi A, Boku N, et al: S-1 monotherapy as second-line treatment for advanced pancreatic cancer after gemcitabine failure. Jpn J Clin Oncol 40: 567-572, 2010.
7. Ueno H, Okusaka T, Ikeda M, Takezako Y and Morizane C: An early phase II study of S-1 in patients with metastatic pancreatic cancer. Oncology 68: 171-178, 2005.

8. Funakoshi A, Senju T and Sumii T: Two cases of advanced pancreatic cancer with cervical lymph node or liver metastasis responding well to S-1 monotherapy. Gan To Kagaku Ryoho 33: 1505-1509, 2006 (In Japanese).

9. Ducreux M, Rougier P, Pignon JP, et al: A randomised trial comparing 5-FU with 5-FU plus cisplatin in advanced pancreatic carcinoma. Ann Oncol 13: 1185-1191, 2002.

10. Rougier P, Zarba J, Ducreux M, et al: Phase II study of cisplatin and 120-hour continuous infusion of 5-fluorouracil in patients with advanced pancreatic adenocarcinoma. Ann Oncol 4: 333-336, 1993.

11. Therasse P, Arbuck SG, Eisenhauer EA, et al: New guidelines to evaluate the response to treatment in solid tumors. European Organization for Research and Treatment of Cancer, National Cancer Institute of the United States, National Cancer Institute of Canada. J Natl Cancer Inst 92: 205-216, 2000.

12. Jemal A, Siegel R, Xu J and Ward E: Cancer statistics, 2010. CA Cancer J Clin 60: 277-300, 2010.

13. Conroy T, Desseigne F, Ychou M, et al: FOLFIRINOX versus gemcitabine for metastatic pancreatic cancer. N Engl J Med 364: 1817-1825, 2011.

14. Dahan L, Bonnetain F, Ychou M, et al: Combination 5-fluorouracil, folinic acid and cisplatin (LV5FU2-CDDP) followed by gemcitabine or the reverse sequence in metastatic pancreatic cancer: final results of a randomised strategic phase III trial (FFCD 0301). Gut 59: 1527-1534, 2010.

15. Androulakis N, Syrigos K, Polyzos A, et al: Oxaliplatin for pretreated patients with advanced or metastatic pancreatic cancer: a multicenter phase II study. Cancer Invest 23: 9-12, 2005.

16. Ulrich-Pur H, Raderer M, Verena Kornek G, et al: Irinotecan plus raltitrexed vs raltitrexed alone in patients with gemcitabinepretreated advanced pancreatic adenocarcinoma. Br J Cancer 88: 1180-1184, 2003.

17. Oettle H, Arnold D, Esser M, Huhn D and Riess H: Paclitaxel as weekly second-line therapy in patients with advanced pancreatic carcinoma. Anticancer Drugs 11: 635-658, 2000.

18. Boeck S, Weigang-Köhler K, Fuchs M, et al: Second-line chemotherapy with pemetrexed after gemcitabine failure in patients with advanced pancreatic cancer: a multicenter phase II trial. Ann Oncol 18: 745-751, 2007.

19. Pelzer U, Kubica K, Stieler I, et al: A randomized trial in patients with gemcitabine refractory pancreatic cancer. Final results of the CONKO 003 study. J Clin Oncol 26 (Suppl 15): 4508, 2008.

20. Lubner SJ, Schelman WR, Mulkerin D, Holen KD, Seo S and LoConte NK: Phase II study of oxaliplatin, high-dose capecitabine, and sorafenib in patients with advanced pancreatic cancer. J Clin Oncol 28 (Suppl 15): 4143, 2010.

21. Starling N, Hawkes EA, Chau I, et al: A dose-escalation study of gemcitabine plus oxaliplatin in combination with imatinib in patients with gemcitabine-refractory advanced pancreatic adenocarcinoma. Ann Oncol: Jul. 12, 2011 (E-pub ahead of print).

22. Nakai $Y$, Isayama $H$, Sasaki T, et al: Impact of $S-1$ in patients with gemcitabine-refractory pancreatic cancer in Japan. Jpn J Clin Oncol 40: 774-780, 2010.

23. Alsamarai S, Zergebel C, Zhang J, Furuie T, Urrea PD and Saif MW: Long term survival on S-1 monotherapy in a patient with recurrent stage IV pancreatic cancer. JOP 9: 185-191, 2008.

24. Yoshino T, Fukutomi A and Boku N: Chemotherapy-naïve advanced pancreatic cancer with multiple liver metastases successfully treated by S-1 monotherapy. A case report. Gan To Kagaku Ryoho 33: 1521-1523, 2006 (In Japanese).

25. Koizumi W, Narahara H, Hara T, et al: S-1 plus cisplatin versus S-1 alone for first-line treatment of advanced gastric cancer (SPIRITS trial): a phase III trial. Lancet Oncol 9: 215-221, 2008.

26. Ajani JA, Rodriguez W, Bodoky G, et al: Multicenter phase III comparison of cisplatin/S-1 with cisplatin/infusional fluorouracil in advanced gastric or gastroesophageal adenocarcinoma study: the FLAGS trial. J Clin Oncol 28: 1547-1553, 2010.

27. Togawa A, Yoshitomi $\mathrm{H}$, Ito $\mathrm{H}$, et al: Treatment with an oral fluoropyrimidine, S-1, plus cisplatin in patients who failed postoperative gemcitabine treatment for pancreatic cancer: a pilot study. Int J Clin Oncol 12: 268-273, 2007. 